\title{
Ischemic Non-Healing Skin Wound Model: Cell Death and Wound Healing Mechanisms
}

DOI: $10.17691 / \mathrm{stm} 2018.10 .4 .08$

Received February 19, 2018

E.I. Morgun, PhD Student'; Junior Researcher, Cellular Biology Laboratory²; Junior Researcher,

Regenerative Medicine Department, Research Institute of Translational Medicine ${ }^{3}$;

O.S. Rogovaya, PhD, Researcher, Cellular Biology Laboratory2; Researcher,

Regenerative Medicine Department, Research Institute of Translational Medicine ${ }^{3}$;

E.A. Vorotelyak, MD, DSc, Corresponding Member of the Russian Academy of Sciences;

Head of Cellular Biology Laboratory2; Head of Regenerative Medicine Department,

Research Institute of Translational Medicine ${ }^{3}$; Associate Professor, Department of Cellular Biology and Histology $4^{4}$

${ }^{1}$ Moscow Institute of Physics and Technology (State University), 9 Institutsky per., Dolgoprudny, Moscow region, 141701, Russia;

${ }^{2}$ N.K. Koltsov Institute of Developmental Biology of the Russian Academy of Sciences, 26 Vavilova St., Moscow, 119334, Russia;

${ }^{3}$ Pirogov Russian National Research Medical University, 1 Ostrovityaninov St., Moscow, 117997, Russia;

${ }^{4}$ Lomonosov Moscow State University, 1 Leninskiye Gory, Moscow, 119991, Russia

The aim of the study was to develop an ischemic skin flap model in laboratory mice with the description of necrotic and apoptotic processes in time course, and demonstrate the regenerative characteristics of non-healing skin wound under the conditions of a formed flap.

Materials and Methods. The study involved $20 \mathrm{BALB} / \mathrm{c}$ mice. The first experimental stage describes an ischemic flap on day 1, 2, 3 , and 5 after flap formation using histology, immunofluorescence, morphometry, and TUNEL. At the second stage a non-healing wound was modeled on the flap, and its pathogenesis was histologically compared with an acute wound.

Results. A formed flap showed such histological ischemic features as blood-filled vessels, micro blood clots in blood vessels, coagulation necrosis, migration of inflammatory cells, as well as extravasation, true skin edema and hyperproliferation of epithelial cells. Maximum pathological changes were found on the third postoperative day, and by day 5 the wound partially healed. Morphometry demonstrated a significant increase in blood-filled vessels and the tendency for epithelial layer reduction in a skin flap of experimental animals compared to the control ones. Apoptotic cells were observed on days 1, 2, 3, and 5 of a formed flap. Some areas showed cell clusters around hair follicles. No apoptotic cells were revealed in control animals.

By the moment of complete regeneration of acute wounds, non-healing wounds exhibited no epithelialization and a formed mature cicatrix that enables to interpret the difference in wound healing as significant.

Conclusion. Ischemia causes major pathological skin changes, as well as results in delayed wound healing. A developed non-healing wound model can be used to study wound healing mechanisms and the ways to influence them.

Key words: apoptosis; cell death; skin wound; ischemia; non-healing skin wound; wound regeneration.

\section{Introduction}

A non-healing wound is a common heterogeneous pathology, and related to etiology, wound age and other factors, which have an effect on wound healing [1]. The factors contributing to wound non-healing are poor blood supply, stably increasing blood pressure, systemic diseases, patient's age, and repeated trauma/injury [2]. Such wounds do not easily respond to conservative therapy [3]. The lack of a unified approach to their treatment results from under-investigated regenerative mechanisms in the wounds [2,4].

Currently, there is no described unified standard model of a non-healing wound on a laboratory animal, an adequate human wound. And an in vitro model does not simulate a complex structure of a wound bed including an ischemic one [5]. One of the common pathogenesis modeling method typical for a non-healing wound is the application of full thickness defect on an ischemic skin flap. The technique is easy to use, standardized and enables to model a wound identical to human pathology. Therefore, the aim of the present study was to develop an ischemic animal skin flap able to adequately demonstrate ischemic evidence, however, necrosis development will not be rampant, and will result in no total flap loss within several days [6]. The model enables to study various pathological processes occurring in skin under ischemia, particularly, cell death, as well as

Corresponding author: Elena I. Morgun, e-mail: lady.morgun2016@yandex.ru 
to create a wound that can be considered non-healing, i.e. regenerative processes in it will proceed more slowly compared to those in an acute incised wound of a similar size.

Necrotic and apoptotic processes are known to play a major role in the pathogenesis of a non-healing wound [1,6-8]. Coagulation necrosis is one of chief histological signs of ischemia in skin [9, 10], and despite all advances in medicine today it is still one of the key problems in surgery [6]. Apoptosis is also a significant primary mechanism of cell death in injury (ischemia/ reperfusion), its cascades are induced by reactive oxygen species. In particular, there was determined the capability to influence ASK-1 and NF-KB as targets in therapeutical effect on apoptosis resulting in survival of skin flap cells [11].

The study of cell apoptotic processes can become a basis for new approaches in regenerative medicine, in particular, when searching targets to have an effect on regenerative processes in non-healing ischemic skin wounds.

The aim of the study was to develop an ischemic skin flap model in laboratory mice with the description of necrotic and apoptotic processes in time course, and demonstrate the regenerative characteristics of a nonhealing skin wound under the conditions of a formed flap.

\section{Materials and Methods}

The experiments were carried out on $20 \mathrm{BALB} / \mathrm{c}$ male mice weighing 18-20 g. All procedures with animals were performed under general anesthesia according to order No. 199H "On the Approval of the Rules of Good Laboratory Practice" (Russia, 2016) and International Guiding Principles for Biomedical Research Involving Animals (CIOMS and ICLAS, 2012), while the ethical principles established by the European Convention for the Protection of Vertebrate Animals used for Experimental and Other Scientific Purposes (Strasbourg, 2006). The study was approved by the Ethics Committee of N.K. Koltsov Institute of Developmental Biology of the Russian Academy of Sciences.

We used Veet cream (France) to depilate hair in a surgical area.

An ischemic skin flap was modeled as follows: full- thickness parallel incisions were made on mice skin; the incisions being symmetrical to the spine, $30 \mathrm{~mm}$ long, $10 \mathrm{~mm}$ away from each other; the necessary condition was to cut off all major vessels from the formed flap. Thus, there was a partially isolated skin flap with impaired circulation, subsequently, surgical sutures being put on the flap edges (Figure $1(\mathrm{a})$ ).

A full-thickness circular wound, 5-7 mm in diameter, was made on the ischemic flap center (Figure $1(b)$ ).

An acute ischemia-free wound was modeled by making a full-thickness circular hole, 5-7 $\mathrm{mm}$ in diameter, on an intact animal's back, coinciding the ischemic flap center (Figure 1 (c)).

During the experiment for ischemic flap modeling, biomaterial samples were taken in animals on days 1 , 2,3 , and 5, and in the experiment for wound healing study - on day 14 after surgery.

Pathological processes in the ischemic flap and a skin wound area were studied on histological specimens. Necropsy of ischemic flap fragments was taken from the flap center, the wound area being wholly taken out, followed by biomaterial fixation in $10 \%$ formalin (Biovitrum, Russia).

Histological specimens, $6 \mu \mathrm{m}$ thick, were made on Microm HM 430 (Thermo Scientific, Germany) and hematoxylin and eosin stained. They were studied and photographed using a microscope BZ-9000 (Keyence, Japan).

Cryosections, $10 \mu \mathrm{m}$ thick, were prepared by a standard technique on a thermostatic cooler SM1900 (Leica Microsystems, Germany).

Apoptotic nuclei were revealed by detecting doublestranded DNA rupture by TUNEL (Biotium, USA).

The vessels were imaged by immunofluorescent staining using the antibodies to marker CD31 (ab28364).

The samples were studied by a fluorescent microscope BZ-9000.

The findings were processed using ImageJ and Origin programs. The number of normal and blood-filled vessels in the ischemic flap was counted in 10 fields of vision for each animal. The derma thickness was measured and the number of epithelial layers in the ischemic flap was counted in two positions: in the flap center and in the marginal area on two skin sample sections. Fields of vision and sections for morphometric analysis were randomly chosen.

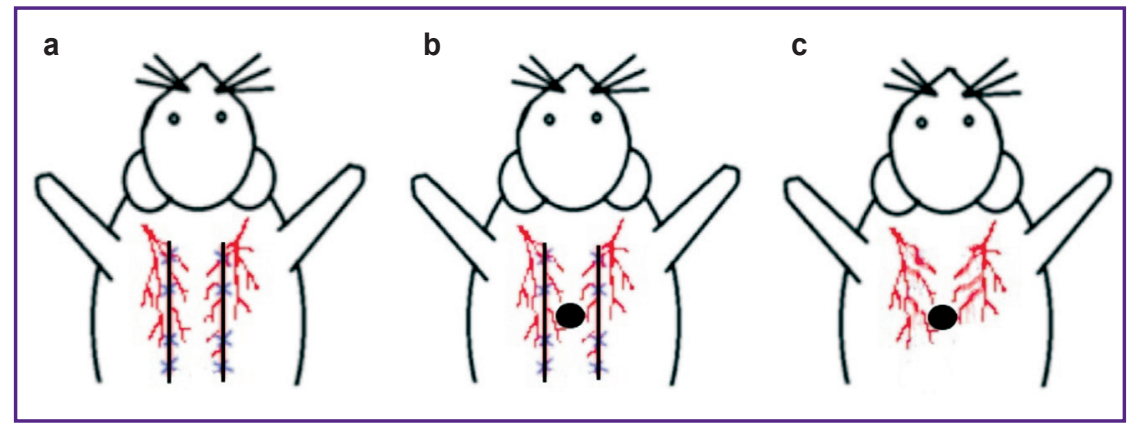

Figure 1. Schematic view of incisions and a circular wound on murine back: (a) in "ischemic flap" group; (b) in "nonhealing wound" group; (c) in "acute wound" group 


\section{Results and Discussion}

The first stage involved the development of $\mathrm{H}$-shaped ischemic skin flap on BALB/c mice. All animals were divided into two groups: an experimental group with "the ischemic flap", and a control group of animals of the same gender and age, without surgery, though depilated in the same way as the experimental group animals.

The second stage consisted of a comparative analysis of a wound process of acute and ischemic wounds (an "acute wound" group and a "non-healing wound" group, respectively).

The animals of "the ischemic flap" group were observed within 5 days after the operation. As early as on the first postoperative day the following changes were revealed: the altered color of the ischemic flap, increasing brown necrotic areas.

All further investigations were carried out on histological samples.

A day after the ischemic flap formation, we observed
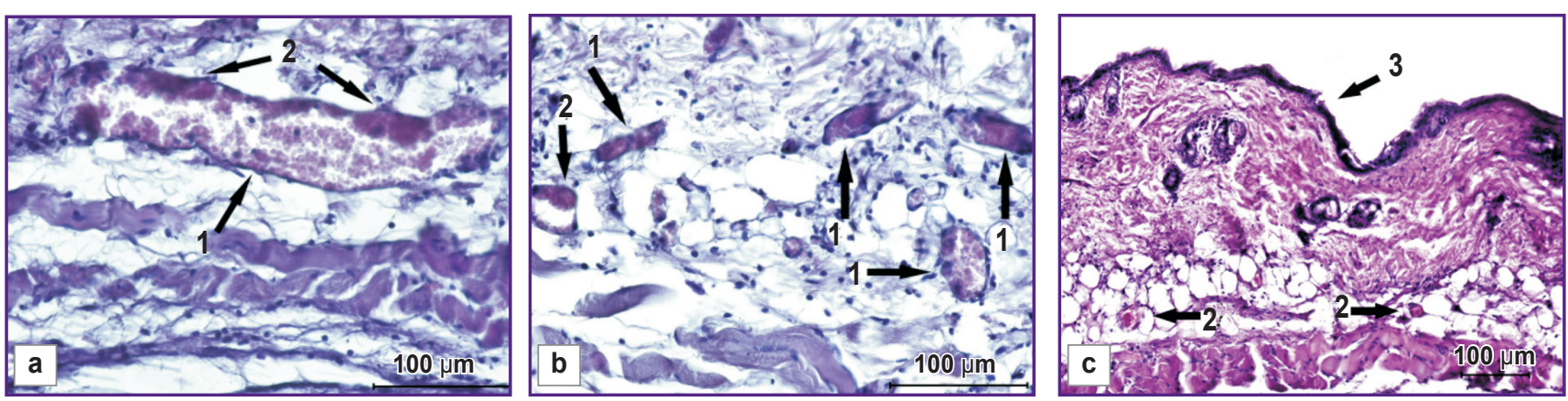

Figure 2. Murine ischemic skin flap area on day 1 after flap formation:

(a) blood-filled vessel, longitudinal section: blood-filled vessel (1), microthrombi in the vessel (2); paraffin section; hematoxylin and eosin staining; x40; (b) blood-filled vessel, cross-section: blood-filled vessel (1), microthrombi in the vessel (2); paraffin section; hematoxylin and eosin staining; x40; (c) pathological changes in derma and epidermis: edema, microthrombi in vessels, epidermal cell death; microthrombi in vessels (2), epidermal cell death (3); paraffin sections; hematoxylin and eosin staining; x20
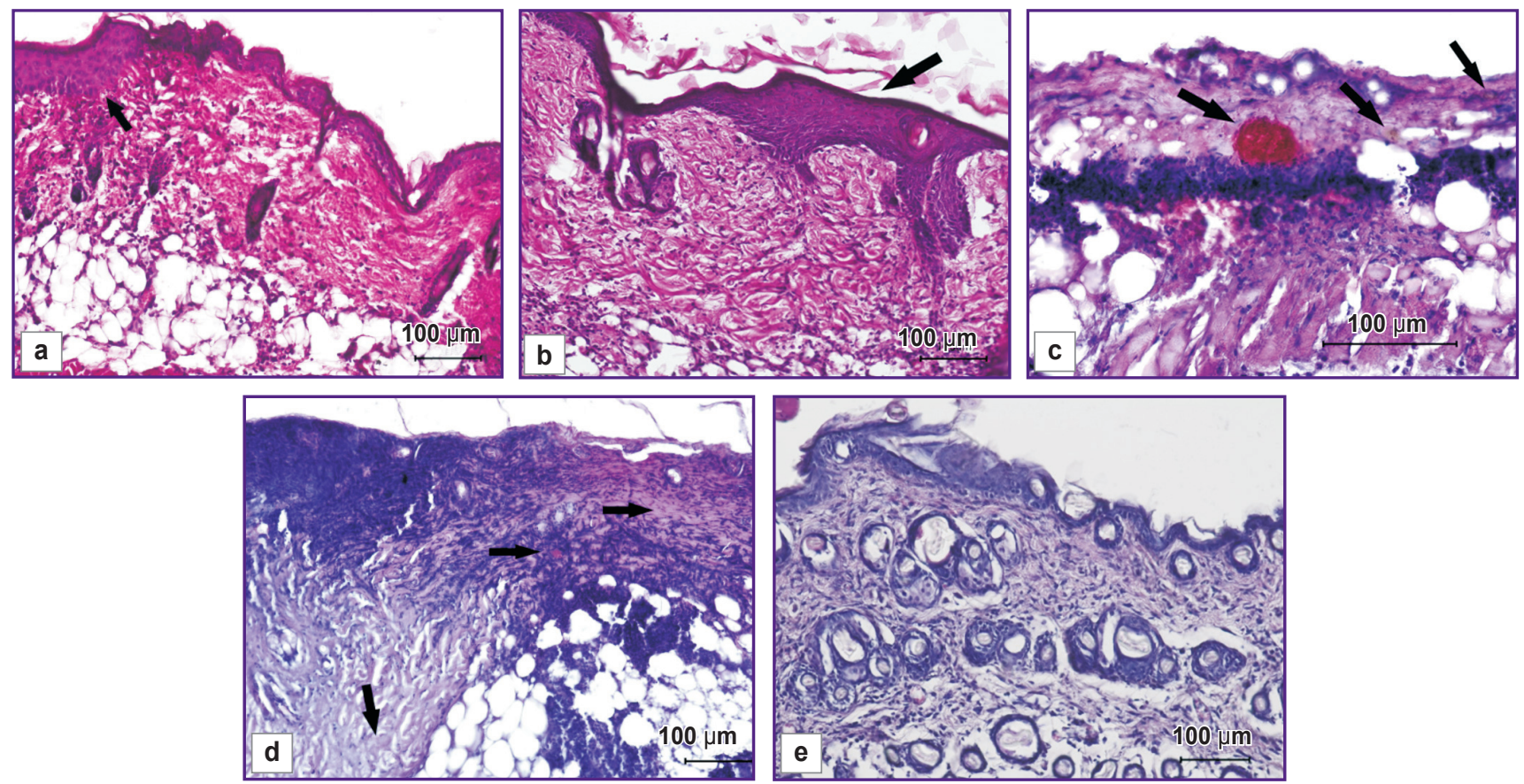

Figure 3. Skin flap area of mice in ischemia and in norm:

(a) hyperproliferation of epidermal cells on day 2 after ischemic flap formation; an arrow indicates keratinocyte hyperproliferation area; paraffin section; hematoxylin and eosin staining; x20; (b) hyperproliferation of epidermal cells on day 5 after ischemic flap formation; an arrow indicates keratinocyte hyperproliferation area; paraffin section; hematoxylin and eosin staining; x20; (c) coagulation necrosis on day 2 after ischemic flap formation; arrows indicate coagulation necrosis areas; paraffin section; hematoxylin and eosin staining; x40; (d) coagulation necrosis on day 5 after ischemic flap formation; arrows indicate coagulation necrosis areas; paraffin section; hematoxylin and eosin staining; x20; (e) a skin flap portion of a control mouse; paraffin section; hematoxylin and eosin staining; $x 20$ 
ischemic signs, in particular, microthrombi in the lumen of derma vessels (Figure 2 (a), (b)).

There were also seen epidermal and dermal cell degradation, edema (Figure 2 (c)), hyperemia and extravasation of vessels (see Figure 2 (a), (b)), the infiltration areas due to inflammatory cells.

In some flap parts there were found pathological changes and lysis of cell nuclei indicating the initial stages of coagulation necrosis.

On day 2 after flap formation, there appeared epithelial areas of hyperproliferation (Figure 3 (a)), and coagulation necrosis and migration of inflammatory cells became marked (Figure 3 (c)).

By day 3 the coagulation necrosis intensity was the highest. The reduction in the number of epithelial layers of the ischemic flap was observed: from the $1^{\text {st }}$ to the $3^{\text {rd }}$, and the increase on day 5 compared to control (see
Figure 3 (a), (b), Figure 4 (a), (b)). On day 5 the skin flap condition on histological specimens was close to healthy skin, however, some animals were found to have vessel extravasation, infiltration of inflammatory cells (Figure 5), epithelial hyperproliferation, edema (see Figure 3 (b), Figure 4 (c), (d)) and necrosis (Figure 3 (d)).

A morphometric analysis showed the tendency for skin flap thickness increase in mice, both in the centre and on the periphery by day 5 that can be related to derma edema (see Figure 4 (c), (d)). The increase in the number of epithelial layers on the $5^{\text {th }}$ postoperative day was due to cell hyperproliferation that was characteristic of wound healing in the norm (see Figure $3(b)$, Figure 4 (a), (b)). However, considering the process duration, it suggests the pathologic character of regeneration.

No degradation signs were found on histological sections of control animals (Figure $3(\mathrm{e})$ ).
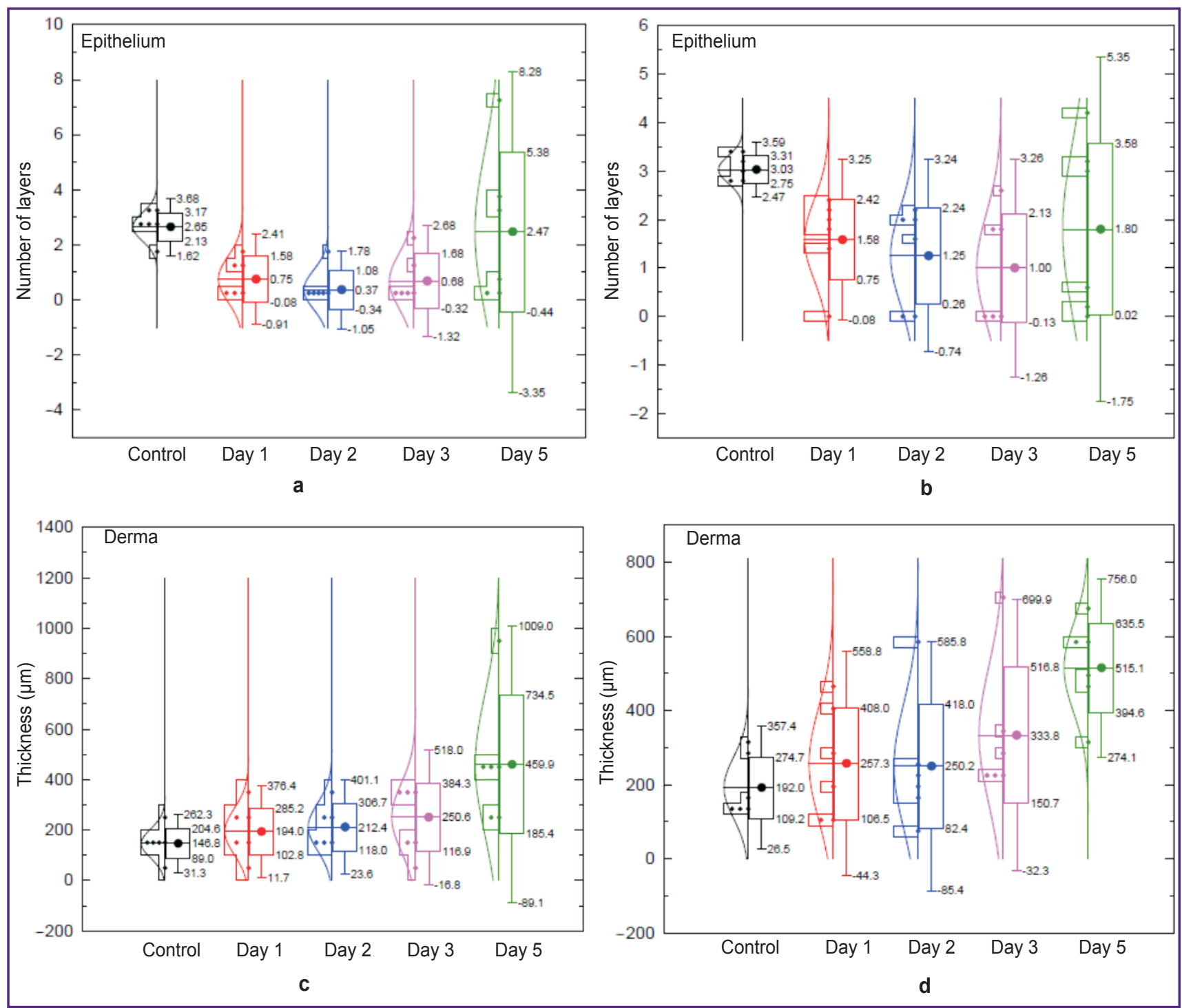

Figure 4. Morphometric analysis of ischemic skin flap at different stages of flap formation:

the number of epithelial layers on the marginal area (a) and in the center (b); derma thickness on the marginal area (c) and in the center (d) 
A portion of blood-filled vessels on histological skin specimens of experimental animals as a possible ischemic effect differed from the control, their number being significantly larger on the $1^{\text {st }}$ postoperative day than later on (Figure 6 (a)). An average number of vessels per field of vision in experimental and control animals were the same (Figure $6(b)$ ).
The study for apoptotic nuclei in the ischemic flap revealed that apoptotic cells in animal epidermis and derma were present in a formed flap on day 1, 2, 3, and 5 after formation. Some areas had accumulation of such cells around hair follicles. No apoptotic cells were found on skin microsections of the control mice.

Coagulation necrosis, infiltration of inflammatory cells,
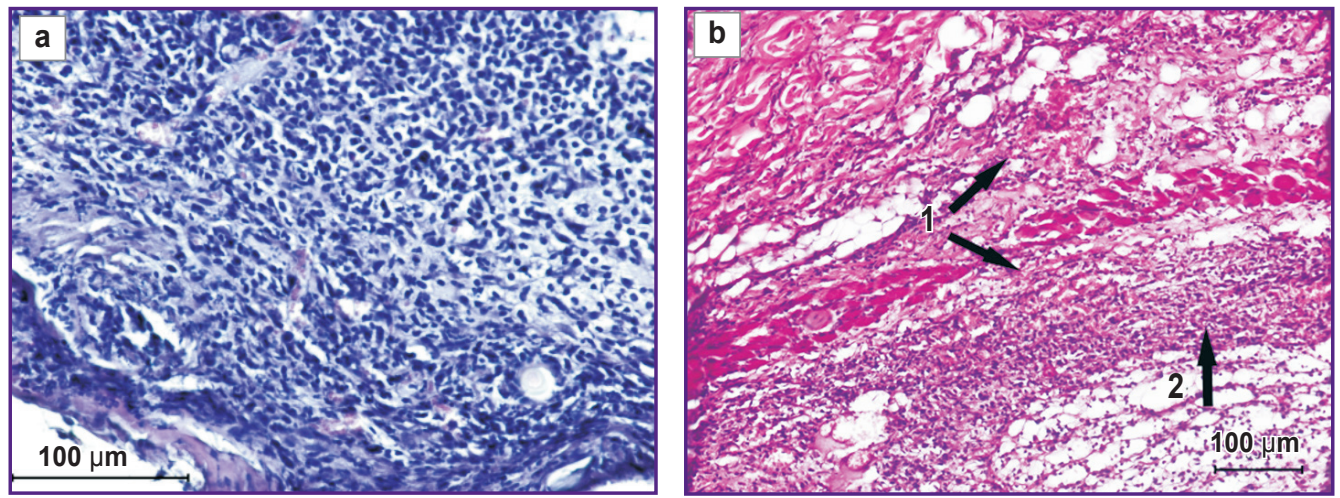

Figure 5. Murine skin flap area on day $\mathbf{5}$ after ischemic flap formation:

(a) infiltration of inflammatory cells; paraffin section; hematoxylin and eosin staining; $x 40$; (b) pathological changes in a skin flap: extravasation of vessels (1), infiltration of inflammatory cells (2); paraffin section; hematoxylin and eosin staining; x20

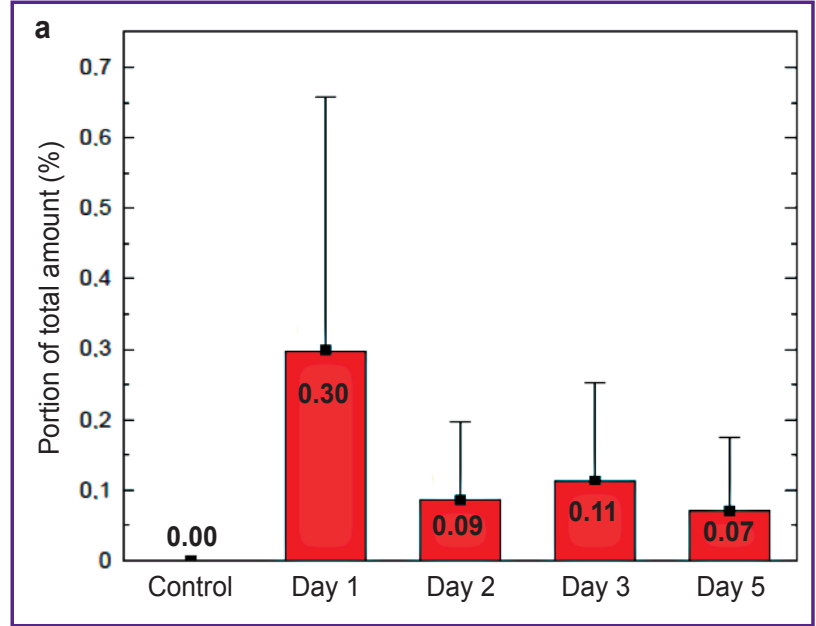

Figure 6. The number of blood-filled vessels in an ischemic skin flap:

(a) a portion of total number per field of vision; (b) average number of vessels per field of vision in an ischemic skin flap; (c) a murine skin flap portion on day 5 after surgery; immunofluorescent detection of vessel epithelial marker CD31 (green staining), DAPI (blue color) stained nuclei; x40
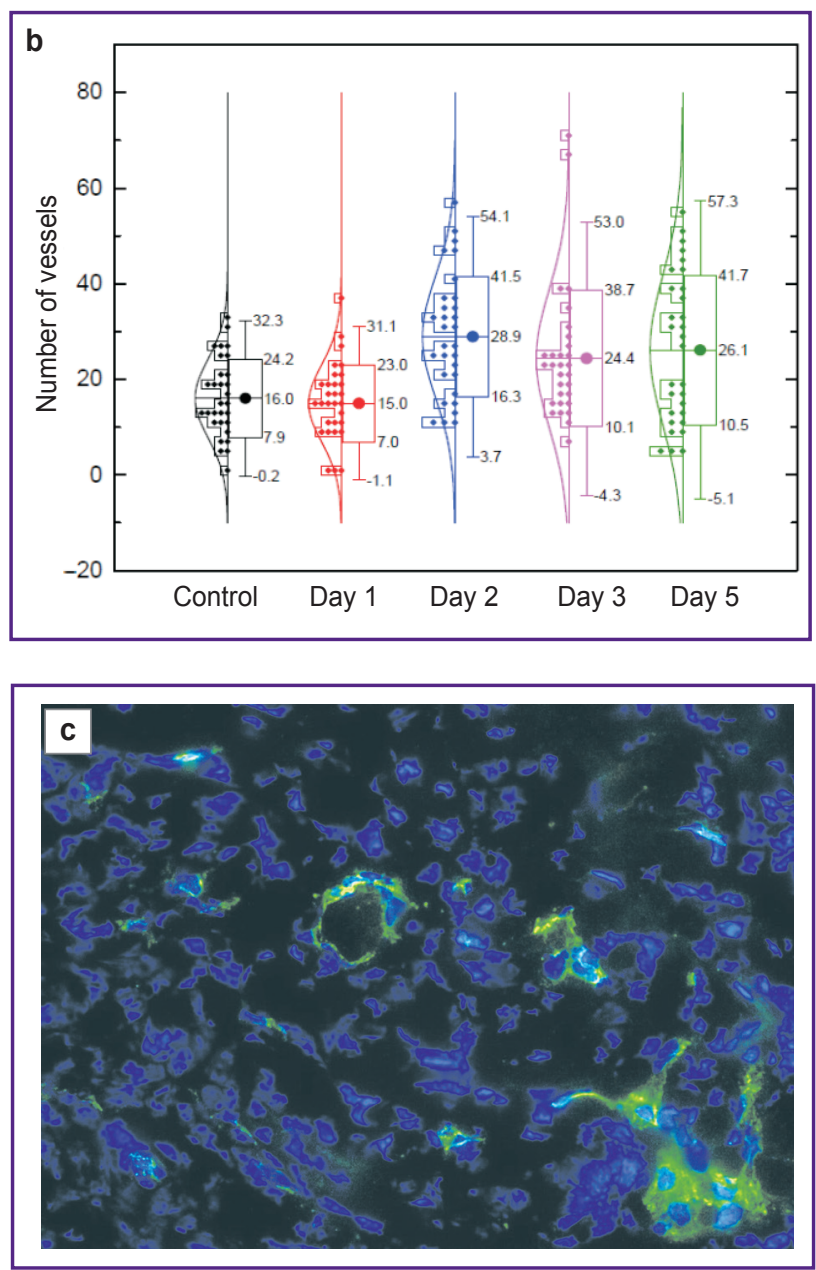


\section{EXPERIMENTAL INVESTIGATIONS}

microthrombi in vessels and their hyperemia are referred to pathomorphological signs of ischemia [9-11]. All the pathological alterations were observed on histological sections of murine skin samples in "the ischemic flap" group that indicated ischemia development within $24 \mathrm{~h}$ after the operation. Subsequently, within a short period of time, due to rather rapid regenerative processes in mice, a flap can regenerate due to vascular invasion on the muscular fascia side. However, the significance of all pathomorphological changes within $24 \mathrm{~h}$ after flap
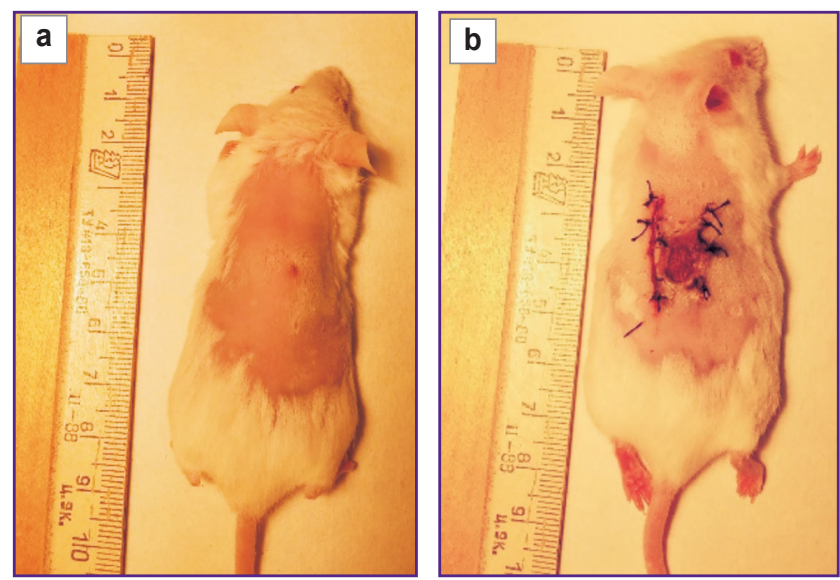

Figure 7. Murine wound condition on day 14 after surgery: (a) from an "acute wound" group; (b) from a "non-healing wound" group formation gives grounds to suggest that the processes occurring in an acute wound in an ischemic skin flap, including regeneration rate, will differ from those in an acute skin wound in mice.

The second stage involved a comparative analysis of regenerative processes in an acute and ischemic skin flap model. The surveillance of the wounds lasted up to 14 days, it can be considered a long-term period of the defect for this animal species. A visual examination of the mice with an acute wound revealed normal wound healing manifested in a reduced wound area and its epithelization by the $14^{\text {th }}$ postoperative day (Figure 7 (a)). By this time the wounds in "non-healing wound" animals were crust-covered (Figure 7 (b)).

Histological examination of wound specimens of mice with an acute wound showed complete closure by normal multilayer keratinizing epithelium, as well as the presence of a formed mature scar in the derma characterized by well organized fibrils and a small number of cells and blood vessels in the wound bed area in mice (Figure 8 (a)). Some specimens had hair follicles in derma indicating the complete recovery of skin structure (Figure 8 (b), (c)).

There was an abundance of fibroblasts in the wound bed center and the lack of intercellular matrix elements in "non-healing wound" group animals. There was no epithelium either (Figure $9(\mathrm{a})$ ). Epithelization and the scar formation could be observed on wound edges (Figure 9 (b)).

Thus, the wounds of "non-healing wound" and
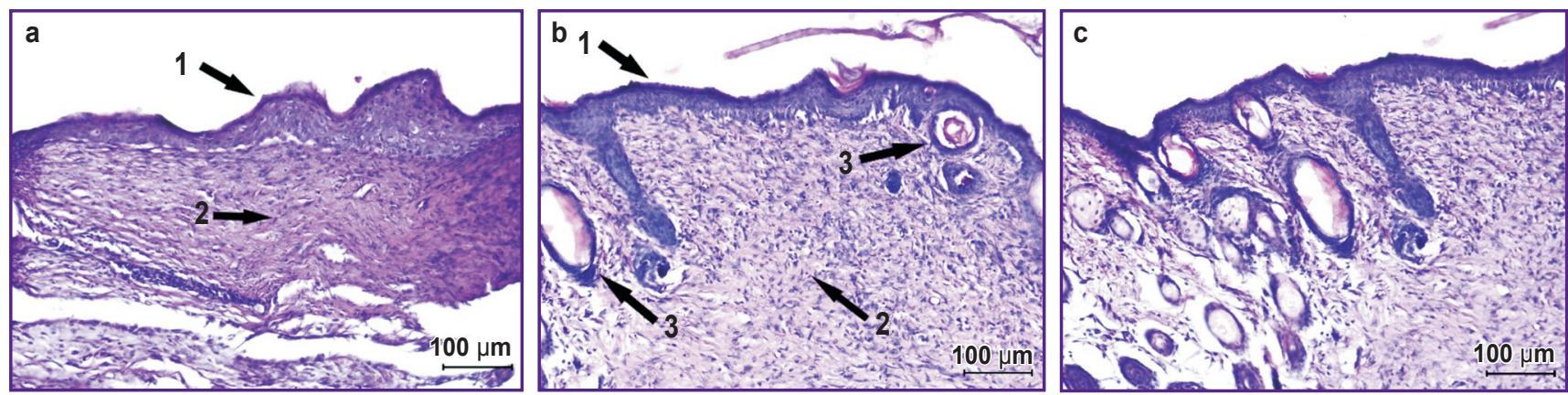

Figure 8. Murine wound from a "non-healing wound" group on day 14 after surgery:

(a), (b) wound center; (c) wound edge; 1 - normal epithelization; 2 - mature scar; 3 - hair follicles in the derma; paraffin section; hematoxylin and eosin staining; $x 20$
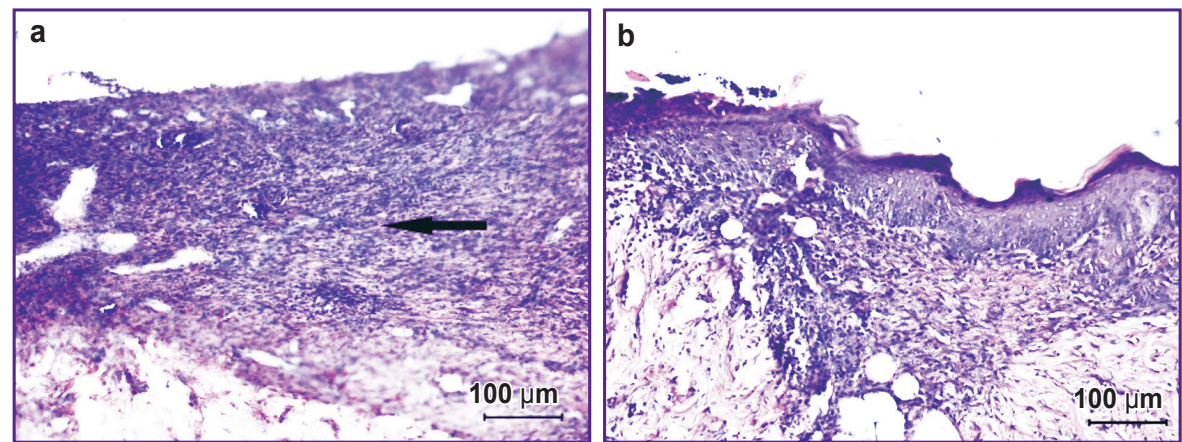

Figure 9. Murine wound from a "non-healing wound" group on day 14 after surgery:

(a) wound center; (b) wound edge an arrow shows the accumulation of fibroblasts; paraffin section: hematoxylin and eosin staining; $x 20$ 
"acute wound" group mice were at different wound healing stages: stage II - a phase of granulation tissue reorganization, formation and maturation, and stage $\mathrm{III}$ - a phase of scar reorganization and epithelization [12]. If complete regeneration of a wound 5-7 $\mathrm{mm}$ in diameter on day 14 is considered the norm for mice, the wounds of mice in a "non-healing wound" group are to be characterized as non-healing due to the lack of epithelization as a wound healing criterion [13].

\section{Conclusion}

The suggested technique of the ischemic flap consists in cutting off all major vessels, so the developed model resembles human pathology. A formed flap has all histological ischemic markers such as necrosis, microthrombi in vessels, their hyperemia, and infiltration of inflammatory cells. These pathological changes persist up to 5 days of an experiment, though no flap necrosis occurs that enables to supervise a wound for a long time. The technique of the ischemic flap is easilyreproducible, and easy to perform. Judging by the characteristics, a developed model can be considered an optimal model of an ischemic skin flap.

The mice in a "non-healing wound" group and those in an "acute wound" group were found to have different wound healing by day 14 after surgery: a non-healing wound was characterized by no epithelization and a mature scar, while an acute wound was completely epithelized. The difference in wound healing duration in mice was assessed as significant, therefore, an obtained non-healing wound can be suggested as a model to study wound healing mechanisms of nonhealing wounds, and to develop possible ways to influence them.

Moreover, the ischemic flap model was observed to show cell death by necrosis and apoptosis. Coagulation necrosis was maximal on day 3 , its signs persisting up to day 5 after surgery. Apoptotic cells were revealed at all experimental stages. The processes can be a key target for therapy using biomedical products.

Funding. The study was carried out within the framework of a research program "Development of manufacturing, storage and application technologies for biomedical cell products for wound healing" according to Grant Agreement with Ministry of Education and Science of the Russian Federation No.14.610.21.0012. Unique identifier is RFMEFI61017X0012.

Conflicts of interest. The authors declare no conflicts of interest related to the present study.

\section{References}

1. Das S., Baker A. Biomaterials and nanotherapeutics for enhancing skin wound healing. Front Bioeng Biotechnol 2016; 4: 82, https://doi.org/10.3389/fbioe.2016.00082.

2. Zhou K., Ma Y., Brogan M.S. Chronic and non-healing wounds: the story of vascular endothelial growth factor. Med Hypotheses 2015; 85(4): 399-404, https://doi.org/10.1016/j. mehy.2015.06.017.

3. Demidova-Rice T., Hamblin M., Herman I. Acute and impaired wound healing: pathophysiology and current methods for drug delivery, part 2: role of growth factors in normal and pathological wound healing: therapeutic potential and methods of delivery. Adv Skin Wound Care 2012; 25(8): 349-370, https:// doi.org/10.1097/01.asw.0000418541.31366.a3.

4. Zhang Q., Chang Q., Cox R.A., Gong X., Gould L.J. Hyperbaric oxygen attenuates apoptosis and decreases inflammation in an ischemic wound model. $J$ Invest Dermatol 2008; 128(8): 2102-2112, https://doi.org/10.1038/jid.2008.53.

5. Gould L., Leong M., Sonstein J., Wilson S. Optimization and validation of an ischemic wound model. Wound Repair Regen 2005; 13(6): 576-582, https://doi.org/10.1111/j.1524475x.2005.00080.x.

6. Lee D., Hong $H_{\text {., }}$ Roh $H_{\text {., }}$ Lee W. The effect of polydeoxyribonucleotide on ischemic rat skin flap survival. Ann Plast Surg 2015; 75(1): 84-90, https://doi.org/10.1097/ sap.0000000000000053.

7. Weinlich R., Oberst A., Beere H., Green D. Necroptosis in development, inflammation and disease. Nat Rev Mol Cell Biol 2016; 18(2): 127-136, https://doi.org/10.1038/nrm.2016.149.

8. Caccamo A., Branca C., Piras I.S., Ferreira E., Huentelman M.J., Liang W.S., Readhead B., Dudley J.T., Spangenberg E.E., Green K., Belfiore R. Necroptosis activation in Alzheimer's disease. Nat Neurosci 2017; 20(9): 1236-1246, https://doi.org/10.1038/nn.4608.

9. Tsai T.C., Tung Y.T., Kuo Y.H., Liao J.W., Tsai H.C., Chong K.Y., Chen H.L., Chen C.M. Anti-inflammatory effects of Antrodia camphorata, a herbal medicine, in a mouse skin ischemia model. J Ethnopharmacol 2015; 159: 113-121, https://doi.org/10.1016/j.jep.2014.11.015.

10. Atlas and synopsis of lever's histopathology of the skin. Edited by Elder D.E., Elenitsas R., Rubin A.I., lofredda M., Miller J., Miller O.F. Lippincott Williams \& Wilkins; 2013.

11. Rah D., Min H., Kim Y., Cheon Y. Effect of plateletrich plasma on ischemia-reperfusion injury in a skin flap mouse model. Int J Med Sci 2017; 14(9): 829-839, https:Ildoi. org/10.7150/ijms.19573.

12. Rany $i$ ranevaya infektsiya WWounds and wound infection]. Pod. red. Kuzina M.I., Kostyuchenok B.M. [Kuzin M.1., Kostyuchenok B.M. (editors)]. Moscow: Meditsina; 1990.

13. Moor A.N., Tummel E., Prather J.L., Jung M., Lopez J.J., Connors S., Gould L.J. Consequences of age on ischemic wound healing in rats: altered antioxidant activity and delayed wound closure. Age 2014; 36(2): 733-748, https://doi. org/10.1007/s11357-014-9617-4. 